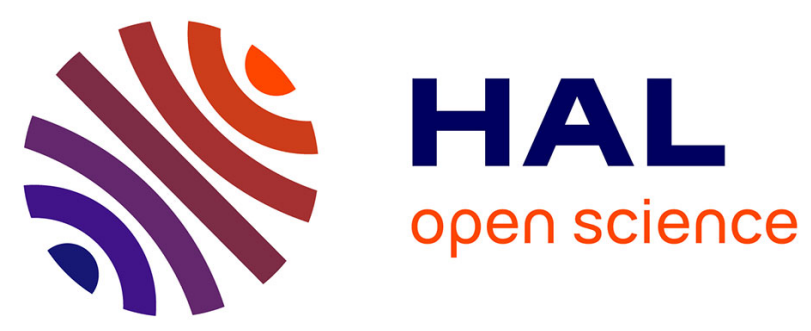

\title{
En réalité : de la modalisation à l'organisation du discours
}

Michel Charolles, Paola Pietrandrea

\section{To cite this version:}

Michel Charolles, Paola Pietrandrea. En réalité: de la modalisation à l'organisation du discours. Travaux de Linguistique: Revue Internationale de Linguistique Française, 2012, 64, pp.111-142. 10.3917/tl.064.0111. halshs-01347373v2

\section{HAL Id: halshs-01347373 \\ https://shs.hal.science/halshs-01347373v2}

Submitted on 21 Jul 2016

HAL is a multi-disciplinary open access archive for the deposit and dissemination of scientific research documents, whether they are published or not. The documents may come from teaching and research institutions in France or abroad, or from public or private research centers.
L'archive ouverte pluridisciplinaire HAL, est destinée au dépôt et à la diffusion de documents scientifiques de niveau recherche, publiés ou non, émanant des établissements d'enseignement et de recherche français ou étrangers, des laboratoires publics ou privés. 


\title{
EN RÉALITÉ : DE LA MODALISATION À L'ORGANISATION DU DISCOURS
}

\author{
Michel Charolles et Paola Pietrandrea \\ De Boeck Supérieur | Travaux de linguistique
}

\author{
2012/1 - n64 \\ pages 111 à 142
}

\section{ISSN 0082-6049}

Article disponible en ligne à l'adresse:

http://www.cairn.info/revue-travaux-de-linguistique-2012-1-page-111.htm

Pour citer cet article :

Charolles Michel et Pietrandrea Paola, «En réalité : de la modalisation à l'organisation du discours », Travaux de linguistique, 2012/1 n64, p. 111-142. DOI : 10.3917/tl.064.0111

Distribution électronique Cairn.info pour De Boeck Supérieur.

(c) De Boeck Supérieur. Tous droits réservés pour tous pays.

La reproduction ou représentation de cet article, notamment par photocopie, n'est autorisée que dans les limites des conditions générales d'utilisation du site ou, le cas échéant, des conditions générales de la licence souscrite par votre établissement. Toute autre reproduction ou représentation, en tout ou partie, sous quelque forme et de quelque manière que ce soit, est interdite sauf accord préalable et écrit de l'éditeur, en dehors des cas prévus par la législation en vigueur en France. II est précisé que son stockage dans une base de données est également interdit. 


\title{
EN RÉALITÉ : DE LA MODALISATION À L'ORGANISATION DU DISCOURS
}

\author{
Michel Charolles ${ }^{*}$ et Paola Pietrandrea ${ }^{* *}$
}

\section{Introduction}

L'étude qui suit ${ }^{1}$ porte sur l'usage de en réalité en français écrit contemporain. Elle est fondée sur un corpus de 150 emplois $^{2}$ extraits et annotés du quotidien Le Monde (novembre-décembre 2000). Dans ces extraits, en réalité invariable et lexicalisé, fonctionne comme une locution prépositionnelle externe à la prédication. Les emplois les plus nombreux sont du type de [1] :

[1] Les enquêteurs ont retrouvé le carton d'emballage et son étiquette informatique, qui tendait à faire croire que le colis avait été posté le 4 août à Gennevilliers (Hauts-de-Seine) et acheminé par la société Federal Express. En réalité, il a été déposé par les expéditeurs le 7 août devant le siège du Syndicat de la Côte-d'Armor et du pays guérandais (Sicapg).

Dans les usages de ce type, en réalité détaché en tête de phrase, signale que le contenu de celle-ci s'oppose à celui d'une ou plusieurs phrases mentionnée(s) précédemment. L'opposition porte sur la valeur de vérité des situations dénotées par les phrases : en réalité indique que la situation à laquelle fait allusion la phrase en tête de laquelle il figure est avérée, au contraire de celles mentionnées dans la complétive qui précède qui ne sont vraies qu'en apparence, comme signalé de façon anticipée, par le segment qui tendrait à faire croire.

Les emplois du type de [1], dans lesquels en réalité fonctionne comme une sorte de connecteur, ont fait l'objet de plusieurs études que nous allons rapidement passer en revue dans la seconde partie. Avant

Université de Paris 3, UMR LATTICE ENS Paris

Université de Roma 3, UMR LATTICE ENS Paris 
d'aborder le fonctionnement de en réalité à l'échelle du discours, il importe cependant, comme nous le montrons dans la troisième partie, de prendre en compte le fait que si tous les emplois de en réalité sont, dans notre corpus, des ajouts syntaxiques, tous ne le sont pas au même degré : certains sont plus intégrés dans leur phrase d'accueil que d'autres. Ces différences se traduisent, comme on le verra, par des différences interprétatives importantes. Dans tous les cas en réalité garde un sens évidentiel (cf. §3.2) mais dans les emplois les plus intégrés, notamment dans les phrases attributives, il fonctionne comme un modalisateur de l'énoncé et ne porte que sur celui-ci, au contraire des emplois dans lesquels il fonctionne comme un marqueur de discours et où il a besoin d'un contexte précédent. Concernant les emplois comme marqueurs de discours du type de [1] qui sont les plus représentés dans notre corpus et qui sont les plus avancés sur le chemin de la grammaticalisation, nous montrons dans la quatrième partie

(i) que, même si en réalité se rapproche des connecteurs oppositifs (réfutatifs ou concessifs) et reformulatifs, il peut aussi se rapprocher de connecteurs signalant d'autres relations de discours,

(ii) que sa valeur évidentielle tend à s'éroder, notamment dans les contextes, relativement nombreux, où en réalité figure dans un énoncé introduisant des faits nouveaux ainsi que dans les contextes où il se trouve dans la portée d'un autre marqueur évidentiel,

(iv) que cette tendance est compensée par les usages, bien représentés dans notre corpus, où en réalité fonctionne, en relation avec en apparence, comme un cadratif de l'énonciation, usages dans lesquels sa valeur évidentielle tend au contraire à se maintenir.

Les analyses présentées dans cette étude visent à extrapoler des hypothèses sur l'évolution du sens de en réalité et sur les voies par lesquelles il a pu devenir un marqueur de discours, ce qui ne va pas du tout de soi pour les modalisateurs en particulier évidentiels (cf. 4.1.).

Le corpus sur lequel nous nous fondons est assurément non représentatif et très limité, mais tel qu'il est, avec les annotations que nous lui avons ajoutées, il offre déjà une riche palette d'emplois qui illustre la grande diversité des usages auxquels se prête en réalité en français contemporain. Cette diversité est essentielle pour les objectifs qui sont les nôtres. Les langues évoluent sous la pression des usages, de tous les usages, dans toutes sortes de contextes, en l'occurrence, pour ce qui est de notre corpus, de cotextes. Les usages réels, outre qu'ils mettent parfois en difficulté les classifications admises, obligent, comme on le verra, à introduire de la continuité et de la polyfonctionnalité là où celles-ci fixent des frontières tranchées. 


\section{2. Études sur en réalité}

Le Trésor de La Langue Française (TLF) note que en réalité, « adverbe d'énonciation », soit conteste une proposition précédente $\mathrm{p}$ et lui oppose une proposition $\mathrm{q}$ " présentée comme vraie ou du moins plus conforme à la réalité », soit s'oppose à une « assertion implicite » et signifie « contrairement à ce qu'on dit, à ce qu'on croit, à ce que pourrait laisser supposer la situation ». Le TLF différencie ces emplois (attestés depuis le XV siècle) où en réalité est une locution oppositive «synonyme » de en fait, des emplois de dans la réalité « complément circonstanciel » qui est employé « par opposition à en principe, en théorie ».

Danjou-Flaux (1982) qui offre une description très fouillée de en réalité aussi bien dans les emplois où il est intégré syntaxiquement que de ses emplois en discours, considère que la valeur oppositive constitue le «noyau sémantique » de la locution en réalité, dont tous les emplois, explique-t-elle, « se laissent décrire à partir d'une opposition faisant référence de manière plus ou moins explicite, à la distinction apparence / réalité » (1982 : 118). Elle différencie, comme le TLF, les usages où en réalité est une locution «qui joue un rôle dans la liaison des énoncés et dans leur enchaînement discursif » à ceux de dans la réalité qui est « un syntagme » (i.e. un complément « essentiellement infra-phrastique ») et qui « ne relie rien $\gg(1982: 127)$.

Le fait que en réalité signale une relation d'opposition est également relevé par Charolles (1984) et par Rossari (1990 et 1993). Charolles parle de « rectification », mais Rossari considère que la valeur oppositive de en réalité est une conséquence du fait que la locution a pour fonction d'indiquer que le locuteur/rédacteur revient sur un énoncé précédent pour exprimer son contenu en des termes qu'il estime plus conformes à «l'ordre du réel » (Rossari, 1993 : 154). La coupure avec l'idée d'opposition est encore plus nette dans Rossari (à par.) qui, tout en retenant que en réalité signale une « relation rhétorique » et fonctionne comme un connecteur, le range dans la classe des « connecteurs métadiscursifs », plus précisément, dans la sous-classe des « reformulatifs» (incluant en fait, en fin de compte...), sous-classe qu'elle différencie des connecteurs métadiscursifs de « spécification » (comme à savoir, en clair, en particulier...) et de « changement de topique » (comme au fait, au reste, autrement, d'ailleurs...).

Iordanskaja et Mel'cuk (1995) dans leur étude sur en réalité et en fait différencient (comme Danjou-Flaux) deux types d'emploi de en réalité : l'un dans lequel en réalité non parenthétique modifie le verbe, et l'autre dans lequel il est parenthétique et forme une locution adverbiale qui fonctionne comme un connecteur. Ces emplois, expliquent les auteurs, correspondent à deux lexies différentes, l'une descriptive et l'autre non descriptive. Leur étude porte essentiellement sur les emplois de en réalité 
connecteur qui est une lexie non descriptive (« signalative, locutive et discursive »). Ils montrent que

(i) en réalité, $Q / Q$ ? est employé par l'énonciateur pour signaler que «l'information au sujet de l'état de choses $\alpha$ spécifié par un énoncé précédent $\varepsilon$ ou une croyance $\varepsilon$ qu'on pourrait avoir comme résultat des énoncés précédents est fausse et que $\mathrm{Q} / \mathrm{il}$ veut que la réponse à la question « $\mathrm{Q}$ ? » soit une réfutation de $\varepsilon$, véhiculant une information vraie au sujet de $\alpha, \mathrm{Q} / \mathrm{Q}$ ? ».

(ii) en fait, $Q / Q$ ? est employé par l'énonciateur pour signaler que "l'information au sujet de l'état de choses $\alpha$ spécifié par un énoncé précédent $\varepsilon$ ou une croyance $\varepsilon$ qu'on pourrait avoir comme résultat des énoncés précédents n'est pas tout à fait bonne que Q/ou la réponse à la question « $\mathrm{Q}$ ? » soit une rectification ou une précision de $\varepsilon$, véhiculant une bonne information au sujet de $\alpha, Q / Q$ ? .

L'étude de Danlos (2012) porte sur en réalité et en effet. Comme Iordanskaja et Mel'cuk (1995), elle ne prend en compte que les emplois de en réalité connecteur. Pour dépasser les approximations des études antérieures qui «manipulent des notions comme la prise en charge, l'évidentialité, la reformulation, la correction, la réfutation, etc. », Danlos s'appuie sur les études de sémantique formelle consacrées à la factivité et aux attitudes propositionnelles et sur le système d'annotation de la base FactBank (Sauri et Pustejovsky). L'analyse de Danlos débouche sur une règle explicitant l'instruction interprétative attachée à en réalité qui implique une mise à jour des attitudes propositionnelles. L'auteur différencie les contextes dans lesquels en réalité apparaît suivant que ceux-ci comportent ou non (dans la phrase hôte de en réalité et dans la phrase précédente) un marqueur d'attitude propositionnelle explicitant ou non une source (un référent à qui on peut imputer une attitude). Nous renvoyons à l'article pour le détail et les aspects techniques.

Les études que l'on vient de passer rapidement en revue apportent un grand nombre d'indications sur le fonctionnement de en réalité en français contemporain. Les analyses ont surtout porté sur les emplois de en réalité connecteur. Danlos ainsi que Iordanskaja et Mel'cuk s'en tiennent à ces seuls usages et indiquent explicitement qu'ils laissent de côté les « acceptions » dans lesquelles en réalité non parenthétique porte sur le seul énoncé dans lequel il figure. Dans la suite de ce travail, nous allons au contraire nous intéresser aux liens entre ces deux grandes formes d'usage de en réalité. Les analyses que nous allons présenter s'inscrivent, comme on le relèvera chemin faisant, dans un programme de travail plus général, incluant des études diachroniques, sur la grammaticalisation des locutions en en $N$. Les travaux sur la grammaticalisation, en particulier l'étude de Schwenter et Traugott (2000) sur in fact, ne sont ni présentés ni discutés dans l'étude 
qui suit qui reste purement synchronique et avant tout descriptive. Comme on le verra également, nous partons de l'hypothèse que en réalité est fondamentalement un modal évidentiel, sans présenter, là non plus, toutes les études et les débats sur la question, ce qui nous aurait entraînés trop loin (pour une présentation en français, cf. Barbet et de Saussure éds, 2012 ; voir aussi Coltier, Dendale et De Brabanter éds, 2009, sur la notion de prise en charge en linguistique).

\section{Intégration syntaxique de en réalité dans sa phrase d'accueil}

\subsection{Emplois exophrastiques et endophrastiques de en réalité}

Dans les emplois comme [1] et comme [2] qui suit, en réalité est, dans la terminologie de Guimier (1996), un ajout extraprédicatif exophrastique : il est non intégré et non régi. On peut le supprimer [2a] sans affecter la grammaticalité de sa phrase hôte, il est parenthétique, prosodiquement détaché de la prédication par la ponctuation, et il ne peut être ni focalisé par un adverbial paradigmatisant $[2 \mathrm{~b}]$, ni clivé [2c]:

[2] En apparence, c'est une affaire classique de plainte en diffamation, déposée par Carl Lang, dirigeant du Front national, contre le Réseau Voltaire, une association de promotion des libertés et de la laïcité. En réalité, le jugement rendu, mercredi 6 décembre, par la 17e chambre du tribunal correctionnel de Paris, présidée par Jean-Yves Monfort, contribue à préciser, en le durcissant, le régime de responsabilité des écrits et des propos diffusés sur Internet.

[2a] En apparence, c'est une affaire classique de plainte en diffamation, déposée par Carl Lang, dirigeant du Front national, contre le Réseau Voltaire, une association de promotion des libertés et de la laïcité. Le jugement rendu, mercredi 6 décembre, par la 17e chambre du tribunal correctionnel de Paris, présidée par Jean-Yves Monfort, contribue à préciser, en le durcissant, le régime de responsabilité des écrits et des propos diffusés sur Internet.

[2b] En apparence, c'est une affaire classique de plainte en diffamation, déposée par Carl Lang, dirigeant du Front national, contre le Réseau Voltaire, une association de promotion des libertés et de la laïcité.

* Seulement en réalité, le jugement rendu, mercredi 6 décembre, par la 17e chambre du tribunal correctionnel de Paris, présidée par Jean-Yves Monfort, contribue à préciser, en le durcissant, le régime de responsabilité des écrits et des propos diffusés sur Internet.

[2c] En apparence, c'est une affaire classique de plainte en diffamation, déposée par Carl Lang, dirigeant du Front national, contre le Réseau 
Voltaire, une association de promotion des libertés et de la laïcité.

* C'est en réalité que, le jugement rendu, mercredi 6 décembre, par la 17e chambre du tribunal correctionnel de Paris, présidée par Jean-Yves Monfort, contribue à préciser, en le durcissant, le régime de responsabilité des écrits et des propos diffusés sur Internet.

Autre test plaidant en faveur de son caractère exophrastique : la phrase en tête de laquelle apparaît en réalité pourrait être niée sans qu'il ne tombe sous le coup de la négation :

[2d] En apparence, c'est une affaire classique de plainte en diffamation, déposée par Carl Lang, dirigeant du Front national, contre le Réseau Voltaire, une association de promotion des libertés et de la laïcité. En réalité, le jugement rendu, mercredi 6 décembre, par la $17^{\mathrm{e}}$ chambre du tribunal correctionnel de Paris, présidée par Jean-Yves Monfort, ne contribue pas à préciser, en le durcissant, le régime de responsabilité des écrits et des propos diffusés sur Internet.

Les emplois comme [1] et [2] contrastent avec les emplois comme [3] et [4] dans lesquels en réalité est inséré et non détaché à la suite du verbe être :

[3] Quand on voit la façon dont le premier ministre louvoie sur ce sujet depuis deux ou trois ans, on se rend compte que sa fameuse méthode, dont on nous a tant rebattu les oreilles, n'est en réalité qu'opportunisme et pilotage à vue.

[4] Pour l'heure, sachant que « 4000 moutons britanniques succombent à la tremblante chaque année, nous voulons savoir si certaines de ces bêtes sont en réalité atteintes d'ESB », a dit Sir John.

Dans [3] et [4] en réalité commute avec dans la réalité ${ }^{3}$ et il en va de même avec [5] où il apparaît à la suite du verbe se traduire :

[5] Vendredi 8 décembre, la ministre de l'Emploi et de la Solidarité, Élisabeth Guigou annoncera une revalorisation de 2,2\% du Revenu minimum d'insertion (RMI), de l'Allocation spécifique de solidarité (ASS) et de l'Allocation d'insertion (AI). Comme le rapporte jeudi La Tribune, ce coup de pouce se traduira en réalité par une augmentation de $1 \%$, en plus des $1,2 \%$ déjà prévus par la loi de finances.

Dans [3], [4] et [5], en réalité est plus intégré syntaxiquement que dans [1] et [2] même s'il demeure un ajout, comme on le voit bien avec [3] où la négation ne porte pas sur en réalité qui reste exophrastique. Si l'on nie [5], la négation pourrait, à la limite, porter sur en réalité mais à condition de préciser à quoi la réalité s'oppose : 
[5a] ... ce coup de pouce ne se traduira pas en réalité, mais seulement en apparence, par une augmentation de $1 \%$, en plus des 1,2\% déjà prévus par la loi de finances

Avec dans la réalité, une telle adjonction ne serait pas nécessaire, la négation porterait sur le circonstant [5b], le clivage serait possible [5c], ainsi que l'interrogation [5d], alors qu'avec en réalité [5e] et [5f] sont difficilement acceptables (même en précisant à quoi s'oppose la réalité) :

[5b] ce coup de pouce ne se traduira pas dans la réalité par une augmentation de $1 \%$, en plus des $1,2 \%$ déjà prévus par la loi de finances

[5c] Ce n'est pas dans la réalité que ce coup de pouce se traduira par une augmentation de $1 \%$, en plus des $1,2 \%$ déjà prévus par la loi de finances

[5d] Est-ce que ce coup de pouce se traduira dans la réalité ?

[5e] ? Ce n'est pas en réalité que ce coup de pouce se traduira par une augmentation de $1 \%$, en plus des $1,2 \%$ déjà prévus par la loi de finances, mais seulement en apparence

[5f] ? Est-ce que ce coup de pouce se traduira par une augmentation de $1 \%$, en plus des $1,2 \%$ déjà prévus par la loi de finances en réalité ou seulement en apparence?

Le fait que la négation, la focalisation et l'interrogation ne puissent pas, ou difficilement, porter sur en réalité montrent que en réalité est fondamentalement exophrastique, même dans les emplois où il n'est pas détaché par la ponctuation.

Pour différencier les emplois de en réalité non parenthétiques comme [3] [4] ou [5] où il commute avec dans la réalité, des emplois comme [1] et [2] où il est parenthétique et exophrastique, nous dirons, en reprenant (et en forçant) la terminologie de Guimier, que dans [3], [4] et [5] en réalité est endophrastique afin de marquer que, sur le continuum de l'intégration syntaxique allant des intraprédicatifs aux extraprédicatifs exophrastiques, ils penchent du côté de l'intégration, comme on le voit bien avec [5] où en réalité est quasiment régi par le verbe se traduire.

Dans notre corpus, les emplois exophrastiques de en réalité sont beaucoup plus nombreux que les emplois endophrastiques :

Tableau 1

\begin{tabular}{|l|c|c|}
\hline $\begin{array}{l}\text { Emplois } \\
\text { endophrastiques }\end{array}$ & 16 & $11 \%$ \\
\hline $\begin{array}{l}\text { Emplois } \\
\text { exophrastiques }\end{array}$ & 134 & $89 \%$ \\
\hline Total & 150 & $100 \%$ \\
\hline
\end{tabular}


Pour ce qui est de la position de en réalité dans sa phrase d'accueil, le tableau 2 qui porte sur la totalité des 150 emplois montre que la distribution est relativement équilibrée entre les emplois en tête de phrase et les cas où en réalité est inséré ou en fin de phrase ${ }^{4}$ :

\section{Tableau 2}

\begin{tabular}{|l|c|c|}
\hline En tête de phrase & 85 & $57 \%$ \\
\hline En position insérée ou finale & 65 & $43 \%$ \\
\hline Total & 150 & $100 \%$ \\
\hline
\end{tabular}

Toutefois, si on examine la distribution des différentes réalisations syntaxiques de en réalité dans les deux positions distinguées, on remarque que en réalité endophrastique n'apparaît qu'en position insérée ou en fin de phrase, tandis que en réalité exophrastique présente une plus grande mobilité :

\section{Tableau 3}

\begin{tabular}{|l|l|c|c|}
\hline \multirow{2}{*}{$\begin{array}{l}\text { Ajout } \\
\text { endophrastique }\end{array}$} & En tête de phrase & 0 & $100 \%$ \\
\cline { 2 - 4 } & $\begin{array}{l}\text { En position insérée } \\
\text { ou finale }\end{array}$ & 16 & $100 \%$ \\
\hline & Total & 16 & $63 \%$ \\
\hline \multirow{2}{*}{$\begin{array}{l}\text { Ajout } \\
\text { exophrastique }\end{array}$} & En tête de phrase & 85 & $37 \%$ \\
\cline { 2 - 4 } & $\begin{array}{l}\text { En position insérée } \\
\text { ou finale }\end{array}$ & 49 & $100 \%$ \\
\hline & Total & 134 & \\
\hline
\end{tabular}

Ces données, qui doivent être relativisées vu le faible effectif du corpus, sont intéressantes surtout quand on les compare à ce qui se passe avec dans la réalité dans le même corpus (deux mêmes mois du Monde). Sans entrer dans une étude détaillée de dans la réalité, on notera tout d'abord que dans la réalité est nettement moins attesté que en réalité : 27 emplois dans la totalité du corpus contre 186 occurrences de en réalité. Sur ces 27 emplois, 23 sont intraprédicatifs, insérés, non détachés, et non parenthétiques. Dans la réalité est ajout de verbes comme ancrer, plonger, fonder, se vérifier, se sentir bien, ou de constructions comme ne rien changer. Dans les emplois de ce type, le $\mathrm{SN}$ la réalité peut lui-même régir un complément : (disparaître) dans la réalité du terrain, (se sentir bien) dans la réalité de 
l'entreprise, ce qui est évidemment impossible avec en réalité où le $\mathrm{N}$ n'est pas déterminé. Dans les trois emplois où dans la réalité est détaché, il commute avec en réalité et le contexte présente les mêmes caractéristiques que dans [1] et [2], à savoir qu'il signale une opposition avec une situation mentionnée précédemment. Ce trait apparaît clairement dans :

[6] Renouvelé au rythme des machines qui le peuplent, l'atelier de production idéal apparaît comme un espace totalement anonyme : on lui demande d'être de plain-pied, régulièrement éclairé par les toitures vitrées qui le recouvrent et le moins encombré possible par les retombées de la structure.

Dans la réalité, ce type idéal n'existe pas : l'usine se développe au gré du marché et des politiques financières de l'entreprise, dans les contraintes du site où elle s'est implantée.

\subsection{Fonctions sémantiques de en réalité}

En réalité ajout endophrastique a pour fonction de préciser que le domaine dans lequel se vérifie l'état de choses dénoté par la proposition coïncide avec la réalité, comme on le voit bien dans [7] et [8] :

[7] Certes, ces nouvelles technologies ouvrent de nouveaux espaces aux artistes de l'image, mais il serait présomptueux de leur attribuer plus de pouvoir artistique qu'elles n'en ont en réalité.

[8] Ceux qui ont des budgets plus importants ne sont en réalité guère plus riches que nous, car ils offrent de plus gros salaires, affirme le président délégué, Michel Bérard.

L'emploi de en réalité déclenche une inférence pragmatique : en réalité suppose la référence à un domaine de vérification autre que la réalité (et donc un domaine de perception fautive ou trompeuse) telle qu'une apparence ou une supposition, dans lequel l'état de choses décrit ne serait pas vérifié. Dans [7] la réalité est explicitement comparée avec les souhaits des promoteurs des nouvelles technologies et dans [8] en réalité rectifie une inférence invitée, à savoir que les pays qui ont des budgets plus importants sont riches. Dans [4] répété ci-dessous, en réalité quoique détaché par la ponctuation est endophrastique, la comparaison se fait entre la réalité et le taux indiqué dans la phrase :

[4] L'effort consenti par l'Europe en faveur de la culture est, en réalité, nettement supérieur à ce dérisoire taux de 0,04\% du budget de l'Union.

Le locuteur décrit un état de choses, à savoir que l'effort consenti par l'Europe en faveur de la culture est nettement supérieur au taux « dérisoire » 
de $0,04 \%$ du budget de l'Union, et il précise que cet état de choses est vérifié dans la réalité. Or, il ne devrait pas être pertinent, là non plus, de préciser que c'est dans la réalité qu'un état de choses donné est vérifié (cela devrait être présupposé comme acquis). Cette violation notoire du principe de pertinence (Grice, 1975 ; Sperber et Wilson, 1986) déclenche une inférence contextuelle susceptible d'expliquer l'ajout en incise de en réalité, à savoir qu'il existe un domaine autre que la réalité dans lequel l'état de choses en question n'est pas vérifié.

Le même fonctionnement se retrouve dans les emplois exophrastiques où en réalité ne sert pas à modifier l'état de choses décrit par la prédication, mais fonctionne comme un prédicat sémantique prenant l'assertion pour argument (Nølke, 1990) :

[9] Officiellement, c'est l'union nationale. En réalité, les dirigeants palestiniens sont terrorisés à l'idée d'attentats suicides en Israël.

[10] Vue du Continent, la BBC est souvent considérée comme le temple du rock. En réalité, Griffin, Bernie Andrews, Jimmy Grant, Brian Willey ou Bill Bebb, producteurs des différentes émissions, ont dû batailler pour obtenir le minimum de considération et de moyens techniques nécessaires aux retransmissions.

Dans les emplois endophrastiques aussi bien qu'exophrastiques en réalité sert donc au locuteur à préciser son attitude vis-à-vis de ce qu'il affirme et, en ce sens, on peut dire qu'il constitue un modal (Lyons, 1977 ; Palmer, 1986) qui porte sur la valeur de vérité de la proposition. Traditionnellement, les modaux portant sur la valeur de vérité sont répartis en deux classes : les modaux épistémiques et les modaux évidentiels (Pietrandrea, 2005, entre autres). Les modaux épistémiques précisent l'opinion du locuteur vis-à-vis de la valeur de vérité de la proposition assertée et ils participent au calcul de la vérité de cette proposition :

\section{[11] Peut-être est-il à la maison.}

Les modaux évidentiels précisent la source sur laquelle est fondée la vérité de l'assertion d'une proposition, cette source pouvant être sensorielle [13], « reportive » (discours rapporté, ouï-dire) [13], ou inférentielle [14], cf. Plungian (2001) pour une classification :

[12] D'après ce que j'ai vu, les dirigeants palestiniens sont terrorisés.

[13] D'après ce qu'on m'a dit, les dirigeants palestiniens sont terrorisés.

[14] D'après ce que j’ai pu comprendre, les dirigeants palestiniens sont terrorisés. 
Avec les modaux épistémiques, l'état de choses modalisé est toujours présenté comme non factuel (il s'agit d'un état de choses enraciné non pas dans la réalité mais dans l'opinion du locuteur):

[11a] Peut-être est-il à la maison, mais je n'en suis pas sûr.

[11b] ? Peut-être est-il à la maison, et effectivement il y est.

Les modaux évidentiels ne supposent que par des inférences supprimables la non-factualité de la proposition modalisée. Ils servent à dire « je fonde mon assertion sur telle source », sans que cela implique forcément la non factualité de l'assertion (Lazard, 1991 ; Pietrandrea, 2005 : 33). Les propositions modalisées par des évidentiels peuvent par conséquent être soit non factuelles [14a], soit factuelles [14b] :

[14a] D'après ce que j'ai compris, les dirigeants palestiniens sont terrorisés (mais je n'en suis pas sûr).

[14b] D'après ce que j'ai compris, les dirigeants palestiniens sont terrorisés (et effectivement on m'a confirmé que ....).

Étant donné cette classification des modaux, il n'est pas difficile de définir précisément la fonction de en réalité : il s'agit d'un modal évidentiel localisant la source d'évidence dans la connaissance de la réalité. Dans les emplois de en réalité exophrastique qui sont prototypiques, l'enracinement de l'évidence dans la réalité sert à marquer l'assertion comme factuelle, et il en va de même avec les endophrastiques :

[14c] *En réalité les dirigeants palestiniens sont terrorisés, mais je n'en suis pas sûr

[14d] En réalité, les dirigeants palestiniens sont terrorisés, et effectivement...

[4a] *L'effort consenti par l'Europe en faveur de la culture est, en réalité, nettement supérieur à ce dérisoire taux de $0,04 \%$ du budget de l'Union, mais je n'en suis pas sûr.

[4b] L'effort consenti par l'Europe en faveur de la culture est, en réalité, nettement supérieur à ce dérisoire taux de $0,04 \%$ du budget de l'Union, et effectivement...

\subsection{En réalité : de la phrase au discours}

\subsubsection{En réalité endophrastique}

Dans les emplois endophrastiques, en réalité inséré ou en fin de phrase peut fonctionner de manière autarcique. C'est ce qui se passe dans [4], [7] et [8] mentionnés ci-dessus ainsi que dans [15] qui est compréhensible indépendamment de ce qui précède : 
[15] La plupart des livres qu'on écrit sont en réalité la même histoire.

Dans [15], la simple allusion aux "livres qu'on écrit" suffit pour que, par inférence, en réalité puisse fonctionner : si on écrit des livres, ceux-ci sont réputés différents puisque, précisément, ils sont diffusés comme autant d'ouvrages distincts mais, dit en réalité, si l'on y regarde de plus près, on a tôt fait de s'apercevoir qu'ils ne racontent jamais que la même histoire, sous des habillages différents. Lorsqu'il est inséré dans une phrase attributive en réalité endophrastique peut s'interpréter au sein de celle-ci, il n'a pas besoin d'un contexte précédent. Il peut même apparaître dans un titre. Notre corpus n'offre aucun exemple de ce genre, mais les emplois comme [16] recueilli sur internet ne sont pas rares :

\section{[16] Tribune}

En France, le solde migratoire est en réalité quasiment nul

Par Hervé Le Bras I Démographe I 10/03/2008 I 18H11

(Rue 89)

Dans [16] en réalité force à reconsidérer le $\mathrm{SN}$ le solde migratoire comme émanant d'une source (mal informée) pour laquelle la France connaîtrait un tel phénomène. Il en va de même dans [17], [18] et [19] également relevés sur internet, ainsi que dans [28] analysé par Charolles (1984) :

[17] Le passé et le futur sont en réalité vécus dans le présent.

[18] je viens de découvrir que mon père biologique est en réalité mon oncle (le frère de mon père).

[19] Le bouddhisme est en réalité une branche de l'Hindouisme.

[20] À Roland Garros les places les plus chères sont en réalité gratuites.

\subsubsection{En réalité exophrastique}

En réalité ajout exophrastique conserve, comme on l'a déjà dit, la propriété de en réalité ajout endophrastique de supposer l'existence d'une source évidentielle alternative à la réalité (et donc fautive) justifiant l'assertion de la prédication. Cette source est le plus souvent explicitée dans le contexte immédiatement précédent : sur les 134 occurrences de en réalité exophrastique, 92 (soit $70 \%$ ) font suite à une phrase mentionnant directement ou indirectement une source alternative. Les moyens formels utilisés pour signaler cette source peuvent être :

_ $\quad$ un adverbial marquant une source « reportive » ou inférable :

[21] En apparence, c'est une affaire classique de plainte en diffamation, déposée par Carl Lang, dirigeant du Front national, contre le Réseau Voltaire, une association de promotion des libertés et de la 
laïcité. En réalité, le jugement rendu, mercredi 6 décembre, par la $17^{\mathrm{e}}$ chambre du tribunal correctionnel de Paris, présidée par JeanYves Monfort, contribue à préciser, en le durcissant, le régime de responsabilité des écrits et des propos diffusés sur Internet.

- un verbe, un adjectif ou un nom qui possèdent une évidentialité intrinsèque (inscrite dans leur sémantisme lexical ; Gosselin, 2010) : comme l'adjectif officiel dans [22], le verbe informer dans [23], les noms apparence dans [24] et soi-disant dans [25],

[22] Le chiffre officiel de 30000 unités est souvent cité. En réalité, ces instructions centrales sont fort mal exécutées par les autorités locales, soucieuses de stabilité sociale.

[23] Le site Web a, par erreur, informé les clients que les produits étaient en cours d'expédition alors qu'en réalité ils n'étaient plus en stock.

[24] Trois ruelles plus loin, un jeune homme, épaulé par un saz (luth à long manche et à trois cordes doubles) et un synthétiseur approximatif, chante des chansons d'apparence sentimentale, qui sont en réalité des poèmes politiques de Sabahattin Ali, membre du Parti communiste turc tué en 1944, alors qu'il tentait de passer la frontière bulgare.

[25] ... qui au nom d'une « soi-disant République plurielle, veulent en réalité sacrifier la France républicaine sur l'autel d'une Europe des régions, qui signifierait tout simplement la victoire du marché sur le politique ».

- $\quad$ un marqueur graphique de discours rapporté, tel que les guillemets dans [26], ou un usage autonymique [27] à partir duquel on peut inférer que les termes employés sont des expressions rapportées :

[26] Sous le titre «Affaire des frégates : Taïwan relance l'enquête », le magazine annonce, vendredi 24 novembre, que « la Chine nationaliste a déposé plainte avec constitution de partie civile » afin d'éclairer les coulisses financières de la vente des navires français, en 1991. En réalité, cette « plainte » n'en est pas une.

[27] Un refuge pour la nuit (qui était en réalité le début du jour).

\subsubsection{Des emplois endophrastiques aux emplois exophrastiques}

L'autarcie dont peuvent faire preuve les en réalité endophrastiques les différencie des en réalité exophrastiques plus nettement que les traits formels (comme le détachement entre virgules) et que les tests syntaxiques exploités en 3.1. Mais, dans un cas comme dans l'autre, en réalité oblige, ainsi qu'on l'a dit, à ajuster soit le contenu de l'énoncé dans lequel il figure 
(emplois endophrastiques), soit celui d'un ou de plusieurs énoncés précédents (emplois exophrastiques) pour que l'instruction évidentielle qu'il encode puisse fonctionner.

La position en tête de phrase impose une interprétation exophrastique dans laquelle en réalité a besoin d'un contexte précédent, mais quand on compare [28] et [28a] la différence n'est pas considérable :

[28] De l'avis général, après les maladresses du début, Mme Clinton a mené une excellente campagne, énergique, minutieuse, cohérente, politique. New York, sa fascination pour les stars et sa légendaire tolérance, était en réalité le tremplin idéal pour elle : «Ici, à partir du moment où on est célèbre, on est new yorkais d'office», explique un politicien local.

[28a] De l'avis général, après les maladresses du début, Mme Clinton a mené une excellente campagne, énergique, minutieuse, cohérente, politique. En réalité, New York, sa fascination pour les stars et sa légendaire tolérance, était le tremplin idéal pour elle : « Ici, à partir du moment où on est célèbre, on est new yorkais d'office », explique un politicien local.

Dans la version originale [28], en réalité amène à considérer le fait que New York soit fasciné par les stars et soit une ville très tolérante comme un facteur défavorable pour la campagne de Mme Clinton, facteur qui s'est finalement avéré un atout. [28a] implique la même inférence mais en lien avec l'évocation de l'avis général dans la phrase précédente, qui ne fait en fin de compte qu'apporter des indications sur la source contre-évidentielle dont en réalité a de toute façon besoin.

Le même raisonnement s'applique en sens inverse. Si on compare [29] avec en réalité exophrastique détaché en position initiale, avec [29a] où il est inséré, la différence n'est à nouveau pas considérable du fait que le contenu de la phrase hôte de en réalité et celui de la phrase précédente facilitent conjointement l'interprétation de en réalité :

[29] Ils sont accusés d'avoir agi contre « la sécurité nationale », ce que tous récusent. En réalité, le « crime » dont ils sont jugés coupables par une justice toujours sous strict contrôle du courant conservateur est la défense des libertés.

[29a] Ils sont accusés d'avoir agi contre « la sécurité nationale », ce que tous récusent. Le « crime» dont ils sont jugés coupables par une justice toujours sous strict contrôle du courant conservateur est en réalité la défense des libertés.

Les emplois comme [28] et [29] sont intéressants pour l'étude de la grammaticalisation de en réalité. En réalité est visiblement bien plus avancé sur ce chemin que dans la réalité. Dans la réalité se prête en effet, comme on 
l'a relevé en 3.1, à des emplois intraprédicatifs, ce qui n'est pas le cas de en réalité. Le $\mathrm{N}$ réalité qui est syncatégorématique a intrinsèquement un sens abstrait, mais en réalité est bien plus abstrait que dans la réalité à cause de l'absence de déterminant et de la préposition en qui a un sens plus qualitatif que locatif (Victorri, 1999). La préposition dans, même quand elle régit un SN dont le $\mathrm{N}$ est abstrait, délimite en effet un intérieur qui s'oppose à un extérieur ou à d'autres intérieurs. En a un sens plus appréciatif lié à l'expérience que nous avons du monde (cf. les praxéologiques de Vigier, 2004, 2005) et à la façon dont nous évaluons ce que nous y faisons ou ce qui s'y passe.

Il n'est donc pas étonnant que en ait donné naissance à des locutions modales comme en vérité (Combettes et Kuyumcuyan, 2007) et en réalité, au contraire de dans qui demeure un localisateur. Dans régit un SN déterminé parce qu'il a besoin d'un référent stabilisé concret (dans une voiture/*dans voiture) ou abstrait (dans la physique quantique/*dans physique quantique) au contraire de en (en voiture, en physique quantique). $\mathrm{Si}$ dans la réalité est possible mais pas dans la vérité c'est sans doute parce que la réalité s'oppose aux apparences, à la fiction... qui délimitent des domaines abstraits, alors que ce n'est visiblement pas le cas avec la vérité 5 . En réalité dénote une attitude, il implique un locuteur ou un énonciateur qui parle de ce qui se passe dans le monde en se fondant sur la connaissance ou l'expérience qu'il en a. D'où sa valeur modale évidentielle. Le $\mathrm{N}$ réalité configure un domaine d'évaluation qui contraste avec d'autres, contrairement au $\mathrm{N}$ vérité qui n'évoque aucun domaine auquel il s'opposerait, d'où le fait que en réalité se prête à des emplois dans lesquels il garde un sens proche de dans la réalité. Il est parfaitement normal aussi que les emplois de ce type n'apparaissent que quand en réalité est plus intégré syntaxiquement dans sa phrase d'accueil où il tend à être compris comme une sorte de circonstant spatial abstrait.

Quand il figure en tête de phrase, l'instruction interprétative codée par en réalité prévoyant de reconsidérer un contenu déjà traité ne peut s'exercer sur le contenu de sa phrase hôte dont le lecteur ou l'auditeur n'ont pas encore connaissance. En réalité a besoin d'un contexte précédent. Si le contexte précédent aussi bien que celui de sa phrase hôte fournissent, comme dans [28] et [29], matière à l'application de l'instruction interprétative qu'il encode, il n'y a aucune difficulté. Par contre, quand en réalité est antéposé et que le contenu de sa phrase hôte ne fournit pas matière pour la procédure de réinterprétation qu'il impose, il a absolument besoin d'un contexte précédent, il prend une valeur « anaphorique ». On peut conjecturer que ce sont les emplois de ce type, qui nécessitent absolument l'appui sur un contexte précédent, qui ont favorisé son évolution vers des emplois comme connecteur. Cette évolution une fois consacrée par l'usage, en réalité a pu apparaitre dans d'autres positions, voire sans ponctuation avant ni 
après, ce qui bien évidemment complique le repérage et ne facilite pas la distinction d'avec les emplois où en réalité est endophrastique.

\section{En réalité connecteur}

\subsection{En réalité connecteur oppositif}

En réalité exophrastique a de toute évidence des affinités avec les connecteurs d'opposition (cf. Danjou-Flaux, 1982). Si l'on part, comme nous l'avons fait, de l'hypothèse que en réalité est fondamentalement (et primitivement) un modalisateur évidentiel, cette évolution ne coule pas de source. A priori les modaux, notamment évidentiels, ne sont pas prédestinés à devenir des connecteurs : d'après $S N$, selon $S N$, pour $S N$ (Charolles, 1987 ; Schrepfer-André, 2006), n'ont pas donné naissance à des locutions qui seraient devenues des connecteurs. À première vue, qui est lexicalisé, n'est pas considéré comme un connecteur (Lenepveu, 2010). De même, on ne voit pas du tout comment il serait possible d'envisager que en apparence, dont le sens est pourtant proche de en réalité, puisse être un connecteur.

L'évolution de en réalité vers des emplois comme connecteur oppositif s'explique essentiellement par sa valeur évidentielle particulière (liée au sémantisme du $\mathrm{N}$ réalité et à la préposition en) qui a fait qu'il a pu encoder, d'abord dans les emplois endophrastiques, une instruction interprétative stipulant de réévaluer la vérité du contenu de sa phrase d'accueil. Pour le reste, on peut conjecturer que sa fréquence d'apparition à la suite de mais, ou d'autres marqueurs d'opposition dont témoignent [30] et [31], a fait le reste :

[30] C'est ce que certains pays comme la Malaisie ou le Venezuela voudraient laisser entendre avec des discours teintés de nationalisme et des revendications d'indépendance vis-à-vis du Nord. Mais en réalité, aucun État n'imagine qu'il soit possible et souhaitable de se déconnecter du Nord et il n'existe plus de définition d'un mode non capitaliste de développement. » S'ils réclament la liberté de pouvoir maîtriser leurs politiques de développement, cet espace de liberté est très limité.

[31] Pour ce faire, ils recourent à une sorte de mythe dans lequel ils auraient à défendre leurs nationaux contre un « envahisseur insondable » baptisé pour le coup « Europe » alors qu'en réalité ils sont les seuls protagonistes du drame.

À force d'être employé à la suite de connecteurs oppositifs dans des contextes comme [30] et [31], en réalité aurait fini par signaler à lui seul une relation de discours d'opposition ${ }^{6}$ comme c'est le cas dans [32] et [33] : 
[32] La discussion au Conseil de sécurité a néanmoins été suspendue en fin de semaine dernière, officiellement dans l'attente des résultats des contacts diplomatiques en cours. En réalité, c'est la menace annoncée d'un veto américain qui a entraîné le report des débats-et du vote.

[33] On parle beaucoup, depuis quelques années, du renouveau de la vie monastique. En réalité, si certains monastères, comme celui de Saint-Wandrille en Normandie, connaissent un grand dynamisme, d'autres sont moribonds. Globalement, on peut dire que pour beaucoup d'instituts créés au XIX ${ }^{\mathrm{e}}$ siècle, autour de tâches éducatives, caritatives ou hospitalières, la mort est assurée.

Dans [32] et [33] en réalité commute avec mais sans changement de sens évident, le contexte précédent précisant à quelle source s'opposent les énoncés hôtes. Lorsque ce n'est pas le cas, comme dans [34], en réalité commute sans problème avec mais en réalité, mais pas avec mais (sans la ponctuation) :

[34] Près de 300 magazines sont proposés aux enfants et aux adolescents, si l'on inclut les revues liées à des modes éphémères (Pokémon...), des séries TV, des suppléments d'éditions... En réalité (mais en réalité/*mais), il faut compter une soixantaine de titres réguliers, aux tirages oscillant entre 50000 et 200000 exemplaires, correspondant à des tranches d'âge bien identifiées : avant deux ans, de trois à cinq ans dès l'entrée en maternelle, à partir de six ans pour l'apprentissage de la lecture, puis de huit à onze ans - la découverte de l'autonomie - avant les années collège.

Si dans [34] mais ne peut fonctionner sans en réalité, c'est parce que le premier énoncé n'est pas signalé clairement comme relevant d'une appréhension superficielle de la situation à laquelle il est fait allusion. La subordonnée adverbiale si l'on inclut les revues ... tend en effet à être comprise, en première lecture, comme une précision rajoutée par le rédacteur pour prouver qu'il y a un très grand nombre de revues destinées aux enfants et aux adolescents. Partant de là, on voit bien pourquoi mais seul passerait très mal : comment le rédacteur, après avoir dit et pris en charge le fait qu'il y a bien 300 titres pourrait-il asserter ensuite, et sans contradiction, qu'il n'y en a qu'une soixantaine ? En réalité est nécessaire (avec ou sans mais) pour que l'enchaînement devienne acceptable parce que son occurrence oblige à reconsidérer le contenu du premier énoncé en regard de celui qu'il introduit, à savoir qu'il faut compter une soixantaine de titres réguliers. La précision que ces titres sont réguliers permet, rétroactivement, de porter l'attention sur le fait que les 300 titres mentionnés n'étaient liés qu'à des modes éphémères et donc à comprendre que la concessive ( si l'on inclut ...), loin d'être assumée par le rédacteur, 
signalait au contraire sa réserve ('si l'on en croit les diffuseurs ... 300 titres (mais) en réalité ...).

Dans [35] mais est aussi nettement moins acceptable que en réalité et mais en réalité :

[35] À défaut d'avoir un prénom, il a déjà un surnom : l'« Homme du millénaire».

Les restes fossilisés de cet "ancêtre », les plus anciens retrouvés à ce jour, ont été découverts au Kenya dans le district de Baringo, par une équipe franco-kényane. Présents dans une couche géologique vieille d'au moins 6 millions d'années, ils confèrent au vénérable Ramidus (4,5 millions d'années) et à la célèbre Lucy (3,2 millions d'années) des airs de jeunots. En réalité (mais en réalité $/ *$ mais) ce sont des ossements non pas d'un mais de cinq paléo-primates, mâles et femelles, qui ont été exhumés.

L'opposition porte bien sur le nombre d' " ancêtres » retrouvés mais en réalité est nécessaire du fait, là encore, que le début du texte n'est pas immédiatement compris comme non pris en charge par le locuteur/rédacteur, et comme faisant allusion au discours « grand public ».

\subsection{En réalité connecteur réfutatif ?}

Dans [34] et [35] en réalité figure en tête d'un énoncé qui réfute une ou plusieurs assertions du contexte précédent. Ces emplois confortent l'analyse de Iordanskaja et Mel'cuk (1995). Le fait que en réalité prenne une telle valeur n'a rien d'étonnant : si le rédacteur (ou un énonciateur mentionné) prend soin de signaler que ce qu'il va dire revient sur ce qu'il a dit, en se plaçant du point de vue de la réalité, il est assez normal que ce soit pour contester le contenu de ce qu'il vient de dire. Dans les emplois de ce type, en réalité est proche du mais réfutatif bien connu (Ducrot, 1980) que l'on trouve dans des énoncés comme [36], dont l'interprétation revient d'ailleurs à [36a] :

[36] À Vienne le Danube n'est pas bleu mais jaune.

[36a] À Vienne le Danube n'est pas bleu (comme le dit la chanson) mais jaune (comme l'enseigne la réalité).

Les emplois réfutatifs de en réalité comme [34] et [35] sont bien attestés dans notre corpus mais, dans certains cas, il est assez difficile de parler de réfutation. Dans [37] par exemple, le fait que le confucianisme serve les intérêts de la classe dirigeante, n'est pas incompatible avec le fait qu'il ait pu favoriser, au moins en partie (cf. le surtout), le développement des pays mentionnés :

[37] Longtemps perçu comme un obstacle à la modernisation, le confucianisme est apparu, dans les années 1980, comme le moteur d'un 
développement propre à l'Asie. En réalité, il sert surtout les fins des dirigeants autoritaires de Singapour, de Pékin ou de Séoul.

Le cas est encore plus net dans [38] et [39] où en réalité n'introduit pas un énoncé réfutant les faits mentionnés dans les deux phrases précédentes :

[38] Les avocats considèrent que c'est un travail d'assistance sociale, pas de juristes. En réalité, la demande de droit des exclus vient souvent des travailleurs sociaux, mais il y a un problème de contact entre les deux mondes.

[39] Comme la période est favorable, beaucoup viennent nous voir la veille d'un entretien pour savoir combien ils peuvent demander et s'ils doivent absolument obtenir le statut cadre, explique-t-il. En réalité, ils n'ont que rarement un véritable projet professionnel.

Dans [37], [38] et [39] en réalité commute avec le mais concessif non réfutatif, également bien connu, que l'on trouve dans des exemples comme [40] qui ne nie pas que les entretiens mentionnés aient été amicaux mais une inférence que l'on pourrait en tirer :

[40] Les entretiens ont été francs mais amicaux. (Ducrot, 1980)

Dans [37] et [38] qui font allusion à un point de vue [37] ou un discours autre [38] en réalité garde son sens évidentiel, quoique la phrase en tête de laquelle il apparaisse fasse allusion à un fait nouveau. Dans [39], où on ne trouve plus aucun indice du même type, en réalité équivaut à un mais concessif, il a perdu une bonne partie de son sens évidentiel.

\subsection{En réalité connecteur reformulatif ?}

L'analyse de en réalité comme connecteur «métadiscursif reformulatif » défendue par Rossari (1990, 1993 et à par.) va dans le sens contraire de celle de Iordanskaja \& Mel'cuk (1995) qui poussent au maximum sa valeur oppositive, notamment pour la différencier de celle de en fait. Rossari ne garde de l'instruction interprétative véhiculée par en réalité que l'idée qu'il implique la révision d'une formulation précédente, que celle-ci émane du locuteur/rédacteur lui-même ou d'un énonciateur mentionné.

Cette analyse s'applique assez bien à des emplois comme [41] où en réalité (non réfutatif) relie deux énoncés formellement très proches. Le parallélisme des constructions fait qu'il est facile de reconnaître les deux segments de discours qu'il met en relation, et donc de parler de reformulation :

[41] L'un se plaint de sa belle-fille, l'autre d'avoir mal aux jambes. En réalité ils se plaignent de tout, de leur famille, de leur solitude, des hôpitaux surtout. 
La reformulation n'est pas paraphrastique, comme ce serait le cas avec c'est-à-dire, autrement dit, en d'autres termes. Elle marque, dit Rossari (1990 : 353), une "distanciation" d'avec le contenu du premier énoncé, "distanciation" qu'elle différencie des cas où il y a "récapitulation" (avec en somme, en un mot, bref), "reconsidération" (avec tout bien considéré, tout compte fait, en fin de compte, ...) et "invalidation" (avec enfin). [42] est proche de [41], sauf que le mouvement est plutôt particularisant, alors que dans [41] il est généralisant :

[42] «Le miracle que constitue l'homme dépasse n'importe quel miracle fait par l'homme. » Le corps humain est en réalité miraculeux, il est tissé de nombres et de musique.

Dans [43], on retrouve le même modèle, mais la construction d'ensemble est plus complexe :

[43] Sylvie Lindeperg présente son ouvrage comme «l'emboîtement de deux propositions ». Elle ne se rend pas justice. Son Clio de 5 à 7, titre emprunté à Agnès Varda, et que le prologue justifie au-delà du bon mot, fonctionne en réalité sur trois niveaux. Il s'agit simultanément d'un travail d'historien analysant des documents d'époque, d'épistémologue réfléchissant aux procédures cognitives induites par le matériau étudié et les outils employés, et de philosophe pratiquant la «pensée rhizomique » appelée par les auteurs de Mille plateaux.

Le premier énoncé E1 fait allusion à “deux propositions” : l'expression est marquée comme empruntée et donc comme potentiellement non prise en charge par le rédacteur. E1 est suivi par un énoncé E2 qui réévalue E1 et qui pourrait être précédé par mais en réalité. En réalité quoiqu'inséré est exophrastique car il ne trouve pas matière à fonctionner au sein de E2. Dans [44], qui suit, les deux énoncés reliés par en réalité sont encore formellement très proches, sauf que la situation évoquée (métaphoriquement) par E1 est argumentativement anti-orientée avec celle (tout aussi métaphorique) dénotée par E2. On est très proche de l'invalidation ou de la réfutation comme le montre le fait que en réalité pourrait commuter avec enfin:

[44] Je me suis dit que j'avais enfin mis le pied à l'échelle. En réalité, je m'étais plutôt flanqué l'échelle sur le pied.

\subsection{Emplois non oppositifs de en réalité}

Des observations qui précèdent il ressort que en réalité stipulant de par son sens évidentiel que le locuteur/rédacteur se fonde expressément sur 
la réalité pour énoncer ce qu'il a commencé à dire (emplois endophrastiques) ou va dire (emplois exophrastiques) suffit à déclencher une inférence contextuelle : si le locuteur/rédacteur prend soin de préciser qu'il se réclame de la réalité pour dire ce qu'il a à dire, c'est qu'il a de bonnes raisons de le faire. Parmi ces raisons on trouve naturellement, en premier lieu, le fait que les apparences et les discours sur celles-ci ne plaident pas en faveur de ce qu'il a à dire. On comprend bien, partant de là, comment en réalité a pu, dès les emplois endophrastriques, être lié avec l'idée d'opposition. Lorsque, comme c'est le cas dans les emplois exophrastiques, il relie l'énoncé dans lequel il figure avec un ou plusieurs énoncé(s) précédent(s), il se rapproche des connecteurs oppositifs, où il peut marquer un mouvement réfutatif aussi bien que concessif. Mais il est aussi possible de le rapprocher des connecteurs reformulatifs (notamment « invalidants » comme enfin) en arguant du fait que la révision des contenus, auquel contraint de toute façon en réalité, implique le discours sur les apparences plutôt que les apparences elles-mêmes.

Cela précisé, il faut bien voir que le rapprochement avec les connecteurs d'opposition que l'on observe dans les emplois exophrastiques expose en réalité à un danger : à force d'être employé dans des contextes de ce type, son sens évidentiel risque de s'éroder. Ce risque est déjà bien avéré, comme nous l'avons relevé à propos de [39] et dans une moindre mesure de [37] et [38] ou en réalité équivaut à un mais concessif. Les emplois de ce type dans lesquels en réalité ne contraste pas avec une autre source évidentielle sont relativement nombreux : 40/134, soit $30 \%$.

Dans [45], la fonction évidentielle de en réalité est érodée au profit d'une fonction plus générique de type reformulatif. En réalité peut commuter avec je veux dire ou à vrai dire, pour tout dire et le contenu qu'il introduit, loin de réfuter ni même simplement de reformuler la phrase précédente, vise plutôt à l'expliquer :

[45] « Nous constatons un bridage de l'offre », a expliqué M. Seillière. «En réalité, a-t-il poursuivi, de nombreuses entreprises ne peuvent satisfaire les commandes », en chiffrant à « 800000 le nombre d'offres d'emploi non satisfaites dans le pays actuellement ».

Dans [46], l'énoncé précédant celui contenant en réalité est pris en charge par le rédacteur, ainsi que le montre le verbe savoir qui est factif, en réalité n'est pas réfutatif, ni non plus reformulatif :

[46] C'est même ce rapport ambigu des Européens à la culture qui explique leur immense difficulté à faire une Europe de la culture : ils savent, par une histoire immémoriale, que cette culture les a autant rapprochés que divisés. En réalité il faut être empirique, et agir au cas par cas, comme cela s'est fait pour l'OMC. 
Dans cet exemple on passe du constat (assumé) que les pays européens savent que leurs cultures nationales peuvent aussi bien les unir que les diviser, à une conclusion qui porte sur les politiques à mettre en place dans ce domaine. En réalité ne commute pas avec mais mais plutôt avec partant de là, dans ces conditions. Dans [46] en réalité, tout en faisant le lien avec les énoncés qui précèdent, signale une volonté de couper court à la discussion générale sur les difficultés des Européens dans l'édification d'une culture commune, pour aborder les faits sous un angle différent, et plus crucial.

Dans [47], le contexte faisant allusion aux idéaux proclamés par les jeunes des années 1968 est favorable à l'apparition de en réalité dans la suite et, comme tel est le cas, on devrait s'attendre à ce que l'énoncé dans lequel il apparaît s'oppose aux précédents :

[47] C'était celle de Mai 68, qui avait proclamé « l'imagination au pouvoir ». Mais, pour faire résister ce slogan à l'épreuve du temps et de la réalité, il aurait fallu que cette génération soit portée par une foi, ou au moins par un émerveillement devant la capacité de l'individu, par une volonté de laisser des issues, des marges de manœuvre. » En réalité, nous, leurs enfants, nous nous sommes heurtés à leurs névroses, leur égoïsme, leur narcissisme : ils nous ont fait croire que chacun ne pouvait penser qu'à lui, que, fatalement, finalement, on ne pouvait «qu'en arriver là ».

Le fait que en réalité ne commute pas facilement avec mais ni même avec mais en réalité montre toutefois qu'il n'en va pas exactement ainsi. Le lien avec les énoncés précédents est, comme dans [46], de type consécutif ('comme cette génération n'était pas portée par une foi ... il en a résulté pour nous, leurs enfants ...'). En réalité introduit des informations nouvelles liées au topique de discours en cours, mais qui ne se situent pas dans le prolongement direct du raisonnement qui précède. En réalité marque une volonté de clore la discussion sur les illusions des parents de la génération 68, pour mesurer les effets bien réels de ces illusions sur leurs enfants. Cet effet de rupture, sur un fond de continuité, peut comme dans [46] et [47] initier une séquence portant sur les conséquences de faits mentionnées précédemment, mais elle peut aussi introduire une séquence commentative [48] ou explicative [49] :

[48] A contrario, le site du candidat démocrate est davantage prisé par des femmes (55\%, contre $39 \%)$, des Noirs (4,1\%, contre 1,7 \%) et des personnes actives avec des revenus plus élevés. En réalité, ces chiffres ne sont pas surprenants. Ils ne font que confirmer ce que l'on savait déjà des deux partis, de leurs candidats et des internautes aux États-Unis. »

[49] Conscient de cette interpénétration entre les deux sociétés qu'Israël a, de son propre chef, encouragée sans relâche depuis trois décennies, 
à la fois économiquement et démographiquement (colonies), Ehoud Barak est bien obligé d'admettre qu'en l'état la frontière en question ne peut exister qu'en pointillé. En réalité, le premier ministre est enfermé dans un dilemme dont il ne pourrait sortir que s'il faisait un choix politique résolu qu'aucun gouvernement israélien n'a, pour l'heure, assumé jusqu' au bout : œuvrer à une partition véritable, sur une base égalitaire.

Dans [49] en réalité commute avec car plutôt qu'avec mais. Car peut du reste parfaitement cohabiter avec en réalité comme on le voit dans [50] :

[50] Et notamment celles de Josselin de Rohan, président du groupe RPR du Sénat, qui a qualifié Armand Riberolles et Marc Brisset Foucault de « cosaques » et de « juges justiciers » De telles critiques sont « inadmissibles et dangereuses, car elles masquent en réalité une mise en cause de l'indépendance de la justice », a jugé Ségolène Royal (PS) dans Le Parisien dimanche.

Dans [1] répété ci-dessous en réalité commute à la fois avec mais en réalité et avec car en réalité (en supprimant la ponctuation après) :

[1] Les enquêteurs ont retrouvé le carton d'emballage et son étiquette informatique, qui tendait à faire croire que le colis avait été posté le 4 août à Gennevilliers (Hauts-de-Seine) et acheminé par la société Federal Express. En réalité,/mais en réalité/car en réalité il a été déposé par les expéditeurs le 7 août devant le siège du Syndicat de la Côte-d'Armor et du pays guérandais (Sicapg).

Ce constat, plutôt paradoxal à première vue, s'explique en fait assez bien. Avec mais, en réalité réfute les deux faits dénotés par la complétive, avec car, en réalité justifie la relative et porte sur le marqueur d'attitude propositionnelle (tendait à faire croire) qui est implicatif négatif. Le sens n'est pas le même. Car en réalité (ou puisque en réalité) passerait mieux avec le verbe au plus-que-parfait (il avait été déposé) et l'on comprendrait que [1] vise à reconstituer scrupuleusement le plan d'action des malfaiteurs. Avec le passé composé en réalité est compris comme oppositif, et donc comme équivalant à mais en réalité : en réalité ramène aux faits, non aux intentions (trompeuses) des protagonistes.

Les emplois passés en revue dans cette partie montrent que si en réalité a tendance, de par sa valeur évidentielle, à se rapprocher des connecteurs oppositifs, il peut aussi se rapprocher d'autres connecteurs et exprimer d'autres relations de discours. Les cas de ce type se rencontrent notamment dans les contextes où il introduit des faits nouveaux et où il est plus difficile d'expliquer qu'il implique une révision du contexte précédent. L'existence de ces emplois montre que en réalité en se rapprochant des connecteurs 
oppositifs (ou autres) perd une partie de sa valeur réévaluative, ce qui est un signe de désémantisation.

\subsection{Atténuation de la valeur évidentielle de en réalité}

Le fait que en réalité connecteur puisse, comme on va le voir, figurer dans un énoncé comportant d'autres marqueurs modaux signalant que le locuteur/énonciateur n'assume pas la vérité du contenu propositionnel de la phrase dans laquelle il figure montre également qu'il a perdu une autre partie de sa valeur évidentielle, qui suppose, on le rappelle, un engagement du locuteur/rédacteur sur la vérité de la situation dénotée par sa phrase d'accueil (cf. 3.2).

Les emplois témoignant de cette évolution sont attestés dans notre corpus, ainsi qu'on peut le voir dans [51] et [52], où en réalité porte sur une proposition modalisée respectivement par les modaux « reportifs » si on écoute ses concurrents et c'est ce qu'il laisse entendre :

[51] Sa recette est-elle plus authentique que celle des trois cents artisans qui fabriquent aussi ce gâteau dans le département des Pyrénées Atlantiques ? En réalité, si on écoute ses concurrents, ce Basque qui a beaucoup voyagé a surtout «trouvé un filon pour vendre ce dessert deux fois plus cher que la moyenne » $(60 \mathrm{~F}, 9,15$, le format de $750 \mathrm{~g}$ ). À l'origine, ce gâteau paysan était fait de farine de maïs et de saindoux : pas de quoi en faire une pièce de musée.

[52] Et il ne s'agit pas uniquement d'argent, même si stock options et rémunération sont plusieurs fois évoquées au cours du sondage. En réalité, le futur dirigeant ne se laissera pas dévorer par l'entreprise - en tout cas, c'est ce qu'il laisse entendre -, et cela, à ce stade de responsabilité dans l'entreprise, c'est vraiment nouveau.

Dans [51] et [52] la proposition modalisée par en réalité n'est pas présentée comme factuelle, comme le montre le fait qu'il est possible d'adjoindre à l'énoncé dans lequel figure en réalité une phrase explicitant l'incertitude du locuteur :

[51a] En réalité, si on écoute ses concurrents, ce Basque qui a beaucoup voyagé a surtout «trouvé un filon pour vendre ce dessert deux fois plus cher que la moyenne », mais je n'en suis pas sûr.

[52a] Et il ne s'agit pas uniquement d'argent, même si stock options et rémunération sont plusieurs fois évoquées au cours du sondage. En réalité, le futur dirigeant ne se laissera pas dévorer par l'entreprise - en tout cas, c'est ce qu'il laisse entendre -, quoique je n'en sois pas convaincu.

Le même constat s'impose avec [53] et [54] où en réalité porte sur un énoncé modalisé épistémiquement respectivement par le conditionnel journalistique [54] et le verbe sembler [53] : 
[53] "Les entreprises proposent une offre rétribution qui se compose de la rémunération globale, de tout ce qui peut contribuer à développer les compétences des salariés, d'un environnement de travail de qualité et d'un temps de travail réduit". Tout cela est bel et bien, mais ces intentions des DRH semblent en réalité assez contradictoires avec les préoccupations qu'elles citent, dans cette même enquête, comme prioritaires pour les deux années à venir.

[54] Restent enfin les thérapeutes et prescripteurs, médecins ou praticiens de médecine douce ou parallèle. Dix-neuf d'entre eux sont poursuivis, mais ils seraient en réalité plus de trois mille.

Dans [53] et [54] l'énoncé dans lequel figure en réalité est introduit par un mais, mais en réalité seul, en tête de phrase, pourrait assumer la même fonction :

[53a] "Les entreprises proposent une offre rétribution qui se compose de la rémunération globale, de tout ce qui peut contribuer à développer les compétences des salariés, d'un environnement de travail de qualité et d'un temps de travail réduit". Tout cela est bel et bien. En réalité ces intentions des DRH semblent assez contradictoires avec les préoccupations qu'elles citent, dans cette même enquête, comme prioritaires pour les deux années à venir.

[54a] Restent enfin les thérapeutes et prescripteurs, médecins ou praticiens de médecine douce ou parallèle. Dix-neuf d'entre eux sont poursuivis. En réalité ils seraient plus de trois mille.

Il est possible que le passage à des emplois comme [51] et [52] ait été facilité par la fréquence des usages comme [53] et [54] avec, pour conséquence, une atténuation de la valeur évidentielle de en réalité. Dans [61] et [54], le N réalité n'a plus son sens littéral, dans la mesure où le rédacteur ne se porte pas garant de la vérité du contenu propositionnel de l'énoncé dans lequel il figure, ce qui ne l'empêche pas de garder sa valeur oppositive. Dans [53], le contenu de l'énoncé précédant en réalité fait allusion aux «propositions » des entreprises, lesquelles propositions ne sont pas encore des faits. L'opposition met en cause deux situations avec lesquelles le rédacteur prend ses distances, en réalité signale qu'il croit plus au contenu du second énoncé (sans en être certain) qu'au contenu du premier. Dans [54], le rédacteur ne se distancie pas du contenu de l'énoncé précédant celui avec en réalité, mais seulement du second : les croyances l'emportent sur la réalité. Les cas de ce type, dans lesquels les apparences peuvent être plus décisives que la réalité, ne sont pas rares. Il suffit de penser par exemple à l'âge : l'âge biologique étant caché, l'apparence devient plus patente que la réalité, d'où la possibilité de forger des exemples comme [55] :

[55] Robert n'avait que 30 ans. En réalité il paraissait beaucoup plus âgé. 


\subsection{En réalité connecteur cadratif}

Si en réalité se rapproche des connecteurs, il n'a pas atteint le stade de grammaticalisation de mais ou de car. Mais, car et en réalité ont en commun d'être des ajouts syntaxiques mais en réalité exophrastique reste parenthétique et il peut occuper différentes positions dans sa phrase d'accueil, comme en fait (Charolles et Lamiroy, à par.) et en effet confirmatif (Charolles et Fagard, 2012). Ces différences ont des conséquences importantes pour l'interprétation des relations de discours (Charolles, 2011). Comme en effet confirmatif, en réalité garde un pouvoir cadratif, il peut indexer plusieurs énoncés faisant suite à celui en tête duquel il est détaché. On le voit bien dans l'extrait suivant où en réalité ferme le cadre introduit par en apparence et ouvre un cadre dont la portée s'étend jusqu'à la fin de l'extrait :

[56] En apparence, c'est une affaire classique de plainte en diffamation, déposée par Carl Lang, dirigeant du Front national, contre le Réseau Voltaire, une association de promotion des libertés et de la laïcité. En réalité, le jugement rendu, mercredi 6 décembre, par la 17 e chambre du tribunal correctionnel de Paris, présidée par JeanYves Monfort, contribue à préciser, en le durcissant, le régime de responsabilité des écrits et des propos diffusés sur Internet. Dans un bulletin paru en juin 1999, le Réseau Voltaire avait publié une notice d'information sur M. Lang, «fanatiquement dévoué à Jean-Marie Le Pen, partisan d'une solution armée au problème Mégret ». Ce n'est pas cette allégation publiée noir sur blanc qui a été attaquée, mais sa version « en ligne » diffusée sur le site Web du réseau.

Il en va de même avec [57] où le contraste se fait avec une séquence de discours rapporté (vous dites que) suivi d'objections (or), et où en réalité indexe, comme précédemment, plusieurs phrases :

[57] A la suite de notre article intitulé : « Le chercheur négationniste Serge Thion révoqué du CNRS » (Le Monde du 27 octobre), nous avons reçu de M. Thion la mise au point suivante : Vous dites que mon dossier « entachait depuis longtemps l'image » du CNRS. Mes activités de chercheur spécialisé dans les questions indochinoises ont mérité le satisfecit de tous les rapporteurs qui ont eu à rendre compte de mon travail, depuis trente ans, à la commission de sociologie. Ce que j'écris en dehors du laboratoire relève, éventuellement, de la justice. Or, je n'ai jamais été poursuivi. Vous dites que j'étais considéré « ces derniers temps, par l'administration comme sans affectation ». En réalité, l'administration a dissous prématurément le laboratoire où je travaillais et qui ne voulait pas m'exclure, et a refusé d'entériner mon accueil dans un autre laboratoire, accueil pourtant accepté par la commission dont je dépendais. Je suis entré 
au CNRS en 1971. Mes positions politiques étaient connues. Elles sont demeurées constantes. L'idée de "dérives idéologiques » est sans fondement.

Dans les emplois de ce type, qui sont bien attestés dans notre corpus, en réalité ouvre une nouvelle rubrique de discours tout en gardant une valeur oppositive (clairement réfutative dans [67]). Comme les cadratifs scéniques (Charolles et Prévost éds, 2003 ; Charolles et Péry-Woodley éds, 2005 ; Vigier et Terran éds, 2005) qui sont couramment exploités, notamment à l'écrit, pour structurer les informations textuelles, du fait qu'ils sont à même d'indexer plusieurs propositions, en réalité est amorcé dans un passage précédent et il fonctionne en corrélation avec en apparence. La position initiale favorise évidemment ce genre d'usage où en réalité, tout en participant au contenu propositionnel de l'énoncé dans lequel il figure et en gardant sa valeur évidentielle, acquiert une portée à droite. Cet avantage est d'autant plus remarquable que les autres modalisateurs ne se prêtent pas facilement à une telle extension du fait que, même quand ils apparaissent en tête de phrase, ils restent étroitement attachés à celle-ci soit par l'inversion du sujet [58a] qui bloque leur portée vers l'aval (cf. Fuchs et Fournier, 2003 ; Lahousse, 2003) soit oblige à répéter la forme complétive [58b] :

[58a] Paul ne devrait pas tarder à arriver. Peut-être a-t-il eu un empêchement de dernière minute. *Un patient l'a-t-il appelé.

[58b] Paul ne devrait pas tarder à arriver. Peut-être qu'il a eu un empêchement de dernière minute, qu'un patient l'a appelé/ *un patient l'a appelé.

Dans les emplois comme [56] et [57] en réalité fonctionne comme une sorte de localisateur énonciatif abstrait (Lamiroy et Charolles, à par.). Son usage est compris comme ancrant l'énonciation dans un lieu particulier, certes plus abstrait que l'espace en deux ou n dimensions mais pas si différent que cela, comme on le voit bien dans [59a] et [59b] où à la maison peut, sans difficultés, être remplacé par en réalité :

[59a] Paul est un type assez compliqué. Au labo, il vouvoie sa femme. (Mais) À la maison, ils se tutoient.

[59b] Paul est un type assez compliqué. Au labo, il vouvoie sa femme. (Mais) En réalité, ils se tutoient.

L'emploi de en vérité (Combettes et Kuyumcuyan, 2007) ne serait pas impossible dans le même contexte mais, le $\mathrm{N}$ réalité garde, comme on l'a relevé en 3.3.3., plus d'attaches avec l'espace que le $\mathrm{N}$ vérité, de sorte que en réalité semble mieux à même de se prêter à des usages cadratifs. Il constitue même un excellent 'space builder' avec toutes sortes d'effets sur la référence, comme le montre bien Fauconnier (1984, 1991). 
Dans les emplois comme [56] et [57] la valeur factuelle de en réalité n'est pas affectée, mais il y a un risque que l'exploitation du potentiel cadratif de la locution à des fins d'organisation de l'information discursive, accélère aussi l'érosion de sa valeur modale et conduise à des usages où en réalité ne signifierait plus en réalité mais servirait simplement à négocier un changement partiel de topique de discours. Si cette possibilité existe, on est apparemment encore assez loin, avec en réalité, de ce qui se passe avec en fait (Blumenthal, 1996 ; Charolles et Lamiroy, à par.) qui se prête couramment, notamment en français parlé, à des emplois comme marqueur de changement de topique de discours. On peut toutefois se demander, au vu d'exemples comme [60], si en réalité (qui commuterait sans problème avec en fait) ne prend pas le même chemin :

[60] Le roi Abdallah II a été donner son sang et toute la presse a titré : « Du sang hachémite pour les blessés de Palestine ». En réalité, le palais est inquiet. « Le roi est en harmonie totale avec son peuple. La situation n'est pas dangereuse, mais nous sommes prêts à toute éventualité », dit Taleb El Rifaï, ministre de l'information et étoile montante de la tendance «moderniste » de la monarchie.

\section{Conclusion}

Les emplois de en réalité analysés dans cet article n'offrent bien entendu qu'une vue partielle des usages auxquels cette locution peut se prêter en français contemporain. Comme on l'a souligné en commençant, le corpus limité d'extraits du Monde sur lequel nous nous sommes fondés ne comporte que des attestations dans lesquelles en réalité est utilisé comme une locution extraprédicative. Pour avoir une vue plus complète des emplois de en réalité en français actuel, il faudrait s'appuyer sur un corpus plus large et plus diversifié. Pour comprendre d'où viennent les emplois contemporains, il faudrait aussi se livrer à une étude diachronique, comme l'ont fait Combettes et Kuyumcuyan (2007) ainsi que Féron (2007) sur en vérité. Il serait également très intéressant de savoir, plus largement, comment ont pu se développer autour des $\mathrm{N}$ réalité, vérité et fait (déterminé ou non) tout un ensemble de locutions prépositionnelles en en, dans et à qui, en se lexicalisant et en se grammaticalisant, ont fini par acquérir des valeurs plus ou moins différentes, voire des sens opposés, pendant que d'autres formes, quoique attestées, ne connaissaient pas la même fortune (comme le montrent Combettes et Kuyumcuyan, 2007).

Pour décrire les emplois contemporains de notre corpus, nous sommes partis de l'hypothèse que en réalité avait une valeur modale, en l'occurrence évidentielle. Ce choix est en accord avec Combettes et Kuyumcuyan qui retracent les étapes ayant conduit à la formation, en français des $\mathrm{XVI}^{\mathrm{e}}$ et 
$\mathrm{XVII}{ }^{\mathrm{e}}$ siècles de locutions comme avec vérité, dans la vérité, de vérité, en vérité et à la vérité et étudient ensuite comment ces locutions ont pu, en se détachant de la prédication, devenir des modalisateurs et, en remontant en tête de phrase, se rapprocher progressivement, pour certaines, des connecteurs. Quoique notre corpus n'offre aucune attestation de en réalité comme complément de manière d'un verbe, le fait qu'il se prête en français contemporain à des emplois endophrastiques et exophrastiques suggère une évolution comparable. Les emplois endophrastiques de en réalité sont déjà typiquement et exclusivement modalisateurs. Bien qu'extraprédicatif, en réalité endophrastique reste, comme on l'a montré, étroitement lié à l'énoncé dans lequel il apparaît et au sein duquel il peut le plus souvent commuter avec dans la réalité et occasionnellement avec réellement. Dans les emplois exophrastiques qui sont de loin les plus nombreux dans notre corpus, en réalité garde sa valeur évidentielle et contrastive, mais il acquiert une portée plus large : il porte sur l'ensemble de l'état de choses dénoté par l'énoncé dans lequel il apparaît et oblige, par inférence contextuelle, à mettre en relation cet état de choses avec un ou plusieurs autres états de choses mentionnés précédemment et qu'il présente comme moins conformes à la réalité.

Dans les emplois exophrastiques, en réalité se rapproche des connecteurs oppositifs. Il peut prendre une valeur oppositive forte (réfutative) ou plus faible (reformulative) mais il peut aussi apparaître dans des contextes non oppositifs (notamment justificatifs). Dans les emplois de ce type, en réalité est exposé à des risques d'érosion sémantique, risques qui sont, comme nous l'avons vu, déjà bien avérés. En réalité connecteur gardant un potentiel cadratif, est également très souvent utilisé (au moins à l'écrit) pour structurer l'information discursive en relation avec en apparence, ce qui tend au contraire à favoriser le maintien de son sens évidentiel.

\section{NOTES}

1. Nous remercions Frédérique Mélanie-Becquet pour l'aide qu'elle nous a apportée dans l'annotation du corpus. Nous remercions également les deux relecteurs anonymes pour leurs observations et leurs suggestions de modification, ainsi que L. Danlos (2012) qui cite et discute certains passages de la version soumise.

2. 150 premiers emplois sur un total de 186 , le corpus comptant 5 millions de mots.

3. Dans [4] en réalité commute aussi avec réellement. Comme dans [3] ce n'est pas le cas, et comme dans [5] la commutation est possible, mais modifie le sens, nous ne nous appuyons pas sur ce test dans la suite. Sur réellement voir DanjouFlaux (1982) et Guimier (1996).

4. Les deux positions ne nous semblant pas distinctives, nous ne les différencions pas. 
5. Ce qui, comme le montrent Fagard et Sarda (2009), n'empêche pas dans d'entrer, en français contemporain, dans la composition de SP (y compris lexicalisés) de sens énonciatif et abstrait.

6. Un tel schéma d'évolution est invoqué par Roulet (1990) à propos de après tout pour expliquer que la locution après tout ait pu être considérée comme un connecteur argumentatif (justificatif). Le raisonnement de Roulet est en gros le suivant : partant de l'hypothèse que « dans une séquence de deux actes, si l'articulation n'est pas marquée par un connecteur, la seconde position est par défaut une position d'argument pour l'acte antérieur » (1990:338), et considérant que, dans les séquences comme (a), le second énoncé est « spontanément interprété comme justifiant le premier », Roulet relève que ce lien pourrait parfaitement être explicité par l'adjonction d'un car (mais non d'un donc ou d'un pourtant) comme dans [aa]. Dans ce cas, rien n'empêcherait, poursuit Roulet, d'adjoindre à la suite de car un après tout comme dans [ab], et cette opération, une fois accomplie, il resterait toujours possible d'éliminer car pour arriver tout simplement à [ac] :

[a] Je vais aller me promener. J'en ai assez d'écrire

[aa] Je vais aller me promener, car j'en ai assez d'écrire

[ab] Je vais aller me promener, car, après tout, j'en ai assez d'écrire

[ac] Je vais aller me promener, après tout, j'en ai assez d'écrire

C'est la fréquence des séquences du type de [ac] qui expliquerait, selon Roulet, que après tout ait pu être analysé comme un connecteur justificatif. Le raisonnement est globalement transposable à en réalité à condition d'admettre, contra l'hypothèse de Roulet, que, dans les séquences «E1. E2 » non marquées (i.e. sans connecteur), les relations oppositives (contre-argumentatives dans sa terminologie) puissent être implicitées au même titre que les relations justificatives (argumentatives).

\section{RÉFÉRENCES}

Barbet C. \& de Saussure (éds), 2012, Modalité et évidentialité en français, Langue Française, 173.

Blumenthal P., 1996, «Le connecteur en fait », in Muller C. (éd.), Dépendance et intégration syntaxique, Tubingen, Max Niemeyer Verlag, p. 257-270.

Charolles M., 1984, "En réalité et en fin de compte », in Construction et transformations des objets du discours, Travaux du Centre de Recherches Sémiologiques, 47, p. 85-110.

Charolles M., 1987, «Spécificité et portée des prises en charge en " selon A » », Revue Européenne des Sciences Sociales, Genève, Droz, T XXV, 77, p. 243-271.

Charolles M., 2011, "Les emplois justificatifs de en effet », in Neveu F., Blumenthal B. \& Le Querler N. (éds), Au commencement était le verbe. Syntaxe, sémantique et cognition, Peter Lang, p. 29-52.

Charolles M. \& Prévost S. (éds), 2003, Adverbiaux et topiques, Travaux de linguistique, 47. 
Charolles M. \& Péry-Woodley M-P. (éds.), 2005, « Les adverbiaux cadratifs », Langue Française, 148.

Charolles M. \& Fagard B., 2012, «En effet en français contemporain : de la confirmation à la justification/explication », Le français moderne 80/2, p. 171-197.

Charolles M. \& Lamiroy B., à par., « Des événements aux faits : quelles différences ? Apport des études sur la grammaticalisation ».

Coltier D., Dendale P. \& De Brabanter P. (éds), 2009, La notion de "prise en charge énonciative », Langue Française, 162.

Combettes B. \& Kuyumcuyan A., 2007, « La formation des modalisateurs en français ; le cas des locutions formées sur vérité », Langue Française, 156, p. 76-92.

Danjou-Flaux N., 1982, «Réellement et en réalité. Données lexicographiques et description sémantique », Lexique, 1, p. 110-139.

Danlos L., 2012, «Formalisation de la sémantique des connecteurs en réalité et (et) en effet », Actes du Congrès Mondial de Linguistique Française 2012, Lyon 4-7/07/2012.

Ducrot O., (1980), Les mots du discours, Paris, Minuit.

FAGARD, B. \& SARDA, L., (2009), «Étude diachronique de la préposition dans », in François J. et al., Autour de la préposition, Caen, Presses Universitaires de Caen, p. 225-236.

FAuconnier G., 1984, Espaces mentaux - Aspects de la construction du sens dans les langues naturelles, Paris, Éditions de Minuit.

Fauconnier G., 1991, « Subdivision cognitive », Communication, 53, p. 229-246.

FERON C., 2007, «Les adverbiaux en vérité et à la vérité en moyen français et en français préclassique », in Combettes B. \& Prévost S. (éds), Études sur le changement linguistique en français, Nancy, PUN, p. 145-155.

Fuchs C. \& Fournier N., 2003, « Du rôle cadratif des compléments localisants initiaux selon la position du sujet nominal », Travaux de Linguistique, 47, p. 79-110.

GuIMIER C., 1996, Les adverbes du français. Le cas des adverbes en - ment, Paris, Ophrys.

Gosselin L., 2010, Les modalités en français. La validation des représentations, Amsterdam/New York, Rodopi.

Grice H.P., 1975, trad fr., 1979, «Logique et Conversation », Communications, 30, p. 57-72.

Guimer C., 1996, Les adverbes du français. Le cas des adverbes en -ment. Paris, Ophrys.

Iordanskja L. \& Mel'cuk I., 1995, « Traitement lexicographique de deux connecteurs textuels du français contemporain «en fait » vs «en réalité » », in Bat-Zeev Shyldkrot H. \& Kupferman L. (éds), Tendances récentes en linguistique française et générale, volume dédié à David Gaatone, Amsterdam/ Philadelphia, Benjamins, p. 211-236.

Lahousse K., 2003, « La complexité de la notion de topique et l'inversion du sujet nominal », Travaux de Linguistique, 47, p. 111-136. 
Lamiroy B. \& Charolles M., à par., « Les adverbiaux cadratifs ». Grande Grammaire du Français.

LAZARD G., 1991, « On the Grammaticalization of Evidentiality », Journal of Pragmatics, 33, p. 339-348.

LenePveu V., 2010, « De l'expression du point de vue à l'anticipation textuelle : le rôle de à première vue », Discours, 7. (http://discours.revues.org/)

Lyons J., 1977, Semantics. [2 volumes.] Cambridge, Cambridge University Press.

NøLKe H., 1990, « La classification des adverbes », Langue Française 88, p. 12-27.

Palmer F.R., 1986, Mood and Modality, Cambridge, Cambridge University Press.

Pietrandrea P., 2005, Epistemic Modality. Functional Properties and the Italian System, Amsterdam/Philadelphia, John Benjamins.

Plungian V. A., 2001, « The Place of Evidentiality within the Universal Grammatical Space », Journal of Pragmatics, 33, p. 339-348.

Rossari C., 1990, « Projet pour une typologie des opérations de reformulation », Cahiers de Linguistique Française, 11, p. 345-359.

Rossari C., 1993, Les opérations de reformulation, Berne, Peter Lang.

Rossari C., à par., « Les connecteurs », Grande Grammaire du Français.

Roulet E., 1990, « Et si après tout, ce connecteur pragmatique n'était pas un marqueur d'argument ou de prémisse impliquée ? », Cahiers de Linguistique Française, 11, p. 329-344.

SCHREPFER-ANDRÉ G., 2006, La portée phrastique et textuelle des expressions introductrices de cadres de discours énonciatifs : les SP prépositionnels en «selon X», Thèse de Doctorat, Université de Paris III.

Schwenter S. \& Traugott E., 2000, « Invoking scalarity. The development of in fact », Journal of historical pragmatics, Vol1 (1), p. 7-25.

Sperber D. \& Wilson D., 1986, trad. fr. 1989, La pertinence, Paris, Minuit.

Victorri B., 1999, « Le sens grammatical », Langue Française, 136, p. 85-105.

VIGIER D., 2004, Les groupes prépositionnels en « en $\mathrm{N}$ » : de la phrase au discours, Thèse de Doctorat, Université de Paris III.

Vigier D., 2005, «Les adverbiaux praxéologiques détachés en position initiale et leur portée », Verbum, XXVII, 3, p. 293-312.

VIGIER D. \& TERRAN E. (éds), 2005, Les adverbiaux cadratifs et l'organisation des textes, Verbum, 27, 3. 